\title{
eJRIEPS
}

Ejournal de la recherche sur l'intervention en éducation physique et sport

11 | 2007

Varia

\section{L'interaction langagière formelle et/ou informelle en Education Physique et Sportive en Tunisie}

\author{
Hafsi Bedhioufi et Nabil Gmada
}

\section{OpenEdition}

\section{Journals}

Édition électronique

URL : https://journals.openedition.org/ejrieps/6960

DOI : 10.4000/ejrieps.6960

ISSN : 2105-0821

Éditeur

ELLIADD

Édition imprimée

Pagination : 5-19

\section{Référence électronique}

Hafsi Bedhioufi et Nabil Gmada, «L'interaction langagière formelle et/ou informelle en Education

Physique et Sportive en Tunisie », eJRIEPS [En ligne], 11 | 2007, mis en ligne le 01 janvier 2007,

consulté le 29 octobre 2021. URL : http://journals.openedition.org/ejrieps/6960 ; DOI : https://doi.org/ 10.4000/ejrieps.6960

La revue eJRIEPS est mise à disposition selon les termes de la Creative Commons Attribution 4.0 International License. 
L'interaction langagière formelle et/ou informelle en Education Physique et Sportive en Tunisie

Hafsi Bedhioufi, \& Nabil Gmada

Institut Supérieur du Sport et de l'Education Physique du Kef. Tunisie

\section{Résumé}

Cet article propose une réflexion sur l'interaction langagière en Education Physique en Tunisie. Si l'on veut que l'école soit l'école de la vie, l'institution scolaire doit s'emparer et avec conviction, de cette préoccupation majeure. Au travers de ses injonctions officielles, elle appuie, de façon récurrente, le fait de privilégier le réinvestissement des moyens de communication hors du monde scolaire (langage informel).

La difficulté de l'élève résulte dans la possibilité de comprendre un contenu véhiculé par trois modes de communication verbales différents (le français pour les termes sportifs, l'arabe langue officielle de l'école et le dialecte largement utilisé par l'enseignant et l'élève lors des différentes phases d'interactions langagières).

Le support linguistique utilisé par l'enseignant permet une meilleure communication et ainsi une meilleure compréhension et donc un meilleur apprentissage.

Lors de trois cycles d'apprentissage en gymnastique nous avons essayé d'étudier la pertinence de ces moyens dans l'acte d'enseignement. En fin de compte la démarche didactique, si elle veut organiser l'éducation physique et sportive, doit au préalable s'atteler à étudier l'importance des interactions langagières formelles ou informelles dans l'enseignement de l'éducation physique et sportive en Tunisie. Nous avons pour objectif de décrire les moyens de communication langagière et leurs effets sur les acquisitions des élèves. Nous manifestons également la volonté de produire des connaissances qui pourraient être utiles aux intervenants en éducation physique et sportive dans le contexte tunisien actuel.

L'éducation physique et sportive est une matière d'enseignement dans le système scolaire tunisien. Une matière sous entend qu'elle dispose d'un contenu et d'un moyen de communication entre l'élève et l'enseignant. «Toute personne qui a vécu un minimum de temps dans une école, disaient M. Crahay et D. Lafontaine, sait que la classe est un lieu où le langage domine : les maîtres et les élèves parlent et/ou écrivent, écoutent et/ou 
lisent presque tout le temps. L'essentiel - sinon, la totalité - de l'information communiquée en classe emprunte le canal verbal et la majorité est transmise oralement ». ( Crahay \& Lafontaine, 1986)

Le langage, les gestes et le silence sont des moyens de communication qu'utilise l'enseignant d'éducation physique et sportive dans l'acte d'enseignement. Lors d'une séance d'éducation physique et sportive l'oralité est dominante dans le système enseignement/apprentissage. L'enseignant explique, décrit et corrige. II démontre ou demande aux autres de démontrer. II utilise aussi des croquis et des schémas comme supports didactiques. De son côté, l'élève écoute, assimile et exécute. Le feedback est la dernière étape de la séance où la parole est donnée à l'élève. Le but de l'acte d'enseignement est l'appropriation d'un savoir-faire qui est tributaire de la capacité de l'élève à comprendre. « Ces échanges verbaux enseignants-classe sont reconnus comme un rituel scolaire ayant une double fonction : provoquer la réflexion et le raisonnement des élèves et susciter leur participation orale »(Gal-Petitfaux et Cizeron, 2006, p. 65).

En effet le socio-constructivisme place le langage au centre des processus d'apprentissages. Pour Vygotsky, le langage permet de médiatiser les relations interindividuelles et par-là médiatiser le rapport activité-conscience. "La pensée de l'enfant dépend, dans son développement de la maîtrise des moyens sociaux de la pensée, c'està-dire dépend du langage». «...Le langage, cette capacité spécifiquement humaine, fournit aux enfants des outils sociaux qui les aident à résoudre des tâches difficiles, à surmonter les actes impulsifs, à planifier la solution des problèmes avant de passer à l'exécution et à maîtriser leur propre comportement. Les signes et les mots servent d'abord de moyens de contact social avec autrui» (Vygotsky, L.S. (1985), p.141). Dans le domaine d'éducation gymnique sportive scolaire, Hauw (1992) signale que les conditions d'acquisition des habilités gymniques sont particulières. Les élèves y sont confrontés à une technique sportive complexe, difficile à comprendre et qu'ils doivent réaliser avec peu de matériel et peu de répétitions. L'apport des interactions sociales entre les élèves peut contribuer à l'amélioration de cet apprentissage. En partant de l'approche socio-constructiviste de l'apprentissage en gymnastique, il y a lieu de noter que la communication verbale enseignant/élève est importante. Pour mener cette réflexion, il paraît nécessaire de commencer par la place qu'occupe l'activité gymnique dans le système scolaire tunisien. Dans un deuxième temps, il sera possible de décrire la mise en place du protocole expérimental. Nous ferons ensuite le point sur les résultats statistiques. Pour expliquer ceux-ci une troisième partie proposera une interprétation et une discussion des données, 
ce qui permettra pour finir de souligner l'importance de la langue informelle dans le processus d'apprentissage en éducation physique et sportive.

\section{Quelle valeur accorder à l'activité gymnique ?}

L'enseignement de la gymnastique tire sa référence de son histoire. Plusieurs institutions dont l'école ont servi de support à la pénétration des activités physiques et sportives en Tunisie.

\section{1. Valeur historique}

C'est sur le modèle des associations sportives françaises aux dénominations patriotiquement connotées: Stade gaulois, la Gauloise, Le Cercle français d'escrime, la Patriote que se constituent à partir de 1920 de nombreuses sociétés sportives tunisiennes. II suffit d'énumérer les noms de ces associations pour mesurer l'impact de ces idéologies : La Musulmane, l'Avenir sportif musulman, I'Union sportive musulmane, l'Espérance sportive de Tunis, le Club africain, l'Etoile sportive du Sahel, la Nacirya (la Victorieuse), la Zitouna sport (du nom de la grande université islamique de Tunis), la Sadikya. Jadis, à l'école Sadikya l'activité physique était enseignée au même titre que la physiologie du corps humain ou le français. Dans cette école moderne l'élite tunisienne intègrait la culture physique occidentale. Lors des tournois, la gymnastique de type amorisienne constituait le premier volet de cette culture physique. A la même époque une autre association à caractère culturel et sportif dite la Nacirya ${ }^{1}$ forme de nombreux gymnastes tunisiens et s'attache à répertorier le patrimoine musical andalou ainsi que la danse autochtone. La Musulmane, qui succède à la Nacirya, s'est constituée sur le modèle de celui de la première association française de gymnastique affilée à l'USGF en 1895. Ces deux associations prennent l'initiative de l'organisation de la fête fédérale de gymnastique de Tunis en 1912, en s'inspirant de la fête fédérale de gymnastique d'Alger de 1896. A partir de 1920 de nombreuses associations sportives tunisiennes se sont constituées sur le modèle des associations sportives françaises (Belaid, 1995).

Jusqu'en 1924, le modèle gymnique, issu des traditions militaires et hygiéniques, prédomine dans le milieu scolaire (During, B. 2005). En tant que matière obligatoire (loi française du 27 janvier 1880), la gymnastique est instituée dans les lycées et collèges de Tunisie. Un cadre juridique régissant l'Education Physique prend forme au fil des années, s'inspirant largement de la législation française.

\footnotetext{
${ }^{1}$ Dans ces associations s'exprimaient les premières volontés d'affirmation nationale et identitaire dans le champ sportif. Paradoxale est cette quête identitaire de l'élite tunisienne, faite à la fois d'une volonté de réappropriation de son passé et de l'imitation et de la promotion de la culture occidentale.
} 
Trois dates jalonnent l'institutionnalisation de l'Education Physique en Tunisie :

- Le décret beylical du 2 décembre 1903 qui crée le Conseil de l'Instruction Publique, au sein duquel seront débattues les questions relatives à l'Education Physique scolaire.

- L'arrêté du 2 février 1928 qui correspond à la création d'un comité supérieur consultatif des sports et de la préparation militaire ; ce qui est révélateur de l'importance qu'acquiert l'éducation physique, devenue discipline spécifique et à part entière dans l'enseignement.

- Le décret beylical du 25 juillet 1943 qui fonde le service de la Jeunesse et des Sports, qui demeurera longtemps attaché à la Direction de l'Instruction Publique. C'est à ce nouveau service que l'éducation physique devra son développement jusqu'au lendemain de l'indépendance. Depuis, la gymnastique est devenue une pratique incontournable dans le système scolaire tunisien. Elle est une épreuve obligatoire dans l'examen du « bac sport » et dans les concours d'entrée aux Instituts Supérieurs du Sport et de l'Education Physique.

\section{2. Valeur pédagogique}

La gymnastique est l'activité physique la plus enseignée dans le système scolaire tunisien. Au moins un cycle est programmé par année d'étude. Cet enseignement est couronné par une épreuve nationale appelée "bac sport ». Pour bien préparer cette épreuve, les croquis, les illustrations et les graphiques, proposés par les enseignants, donnent du sens à l'activité d'apprentissage de l'élève. Les échanges langagiers sont importants et le vocabulaire de l'apprenant est enrichi par un vocabulaire spécifique. La mémorisation de la succession des éléments gymniques (notion d'enchaînement) et la liaison des éléments privilégient la continuité et la recherche de l'aspect esthétique. Dans la mesure où la gymnastique et l'athlétisme continuent d'être la base de l'éducation physique, il est clair que l'on peut considérer que l'élève a acquis des connaissances spécifiques importantes en la matière. II paraît logique de proposer à l'élève, au terme de sa scolarité, une gamme d'éléments gymniques existant dans sa programmation officielle.

\section{3. Conduite de l'expérience}

Pour la réalisation de ce travail nous nous sommes successivement confronté aux étapes suivantes.

\section{Etape I}

Sur les 558 élèves de classes terminales nous avons choisi un échantillon de 240 élèves répartis sur les trois lycées de la ville du Kef où les enseignements sont mixtes. Chaque classe dispose de trois séances d'une heure chaque semaine. 


\section{Etape /I}

Les enseignants retenus pour assurer les cycles d'enseignement ont le même niveau de certification et une expérience d'au moins dix ans sans interruption. Ils ne sont pas spécialistes en gymnastique. On a demandé aux enseignants d'utiliser une seule langue lorsqu'ils donnent la parole à la classe pour instaurer un dialogue ou lors de la présentation collective des consignes ou lors du bilan en fin de leçon. Le recueil des données s'est fait à la fin du cycle (deux semaines plus tard). Le contenu des séances est librement choisi par les enseignants afin de respecter la spécificité de chaque classe. Divers éléments gymniques sont imposés lors des séances qui sont : saut vertical + tour complet, saut de l'ange, renversé avant, renversé arrière, renversé arrière avec passage à l'appui tendu renversé, appui tendu renversé (ATR), appui tendu renversé tenu jambes serrées en pont, appui tendu renversé valse demi-tour, rondade saut vertical et souplesse avant ou arrière.

\section{Etape III}

Pour recueillir des données exploitables, nous avons remis aux élèves un tableau qui illustre huit éléments gymniques. Les élèves essaient d'identifier ces derniers, sans imposition de langue car nous croyons que la communication verbale en classe permet d'identifier les différents éléments gymniques et que l'élève est capable de faire le passage de l'oralité à l'écrit. L'appartenance socio-culturelle est un facteur important dans la maîtrise de la langue. Notre intérêt porte sur le contexte social de la cognition. L'approche genre est porteuse de sens. Nous dépassons, dans notre recherche, les oppositions classiques (nature-culture, parole-écriture, masculin-féminin...) pour faire apparaître que les élèves, même s'ils sont en interaction entre eux et avec l'enseignant, ont leur propre rapport au contenu de la leçon. Chacun a des connaissances antérieures susceptibles de favoriser la maîtrise de sa capacité de communication (langue). L'élève tunisien puise dans son appartenance familiale et son vécu des éléments langagiers qui traduisent, a priori, la différence des sexes. Une maîtrise de la langue française est largement attribuée plus aux filles qu'aux garçons. Ces derniers sont influencés par le langage sportif quotidien produit dans les espaces publics qu'ils fréquentent (café, stade, le quartier, les salles de jeu etc). Par une analyse du contenu des commentaires nous essayons de montrer que le choix de la langue d'expression dépasse l'aspect singulier de l'élève. La prise en compte du contexte social et culturel est indispensable. 


\section{Les résultats statistiques}

\section{1. Saut de l'ange}

Les résultats obtenus montrent que le pourcentage du choix du dialecte est de $60,4 \%$. II est de $53,3 \%$ chez les filles et $67,5 \%$ chez les garçons ce qui nous autorise à dire que la langue informelle est la plus choisie par les élèves. Nous pensons que le choix est le résultat d'une connaissance antérieure de l'élément gymnique car le saut de l'ange est un élément qui figure dans le programme des élèves depuis la première année de l'enseignement secondaire.

Tableau I : saut de l'ange

Fréquence

\begin{tabular}{llll}
\hline & Arabe & Français & Dialecte \\
\hline Garçons & 25 & 14 & 81 \\
Filles & 0 & 56 & 64 \\
\hline
\end{tabular}

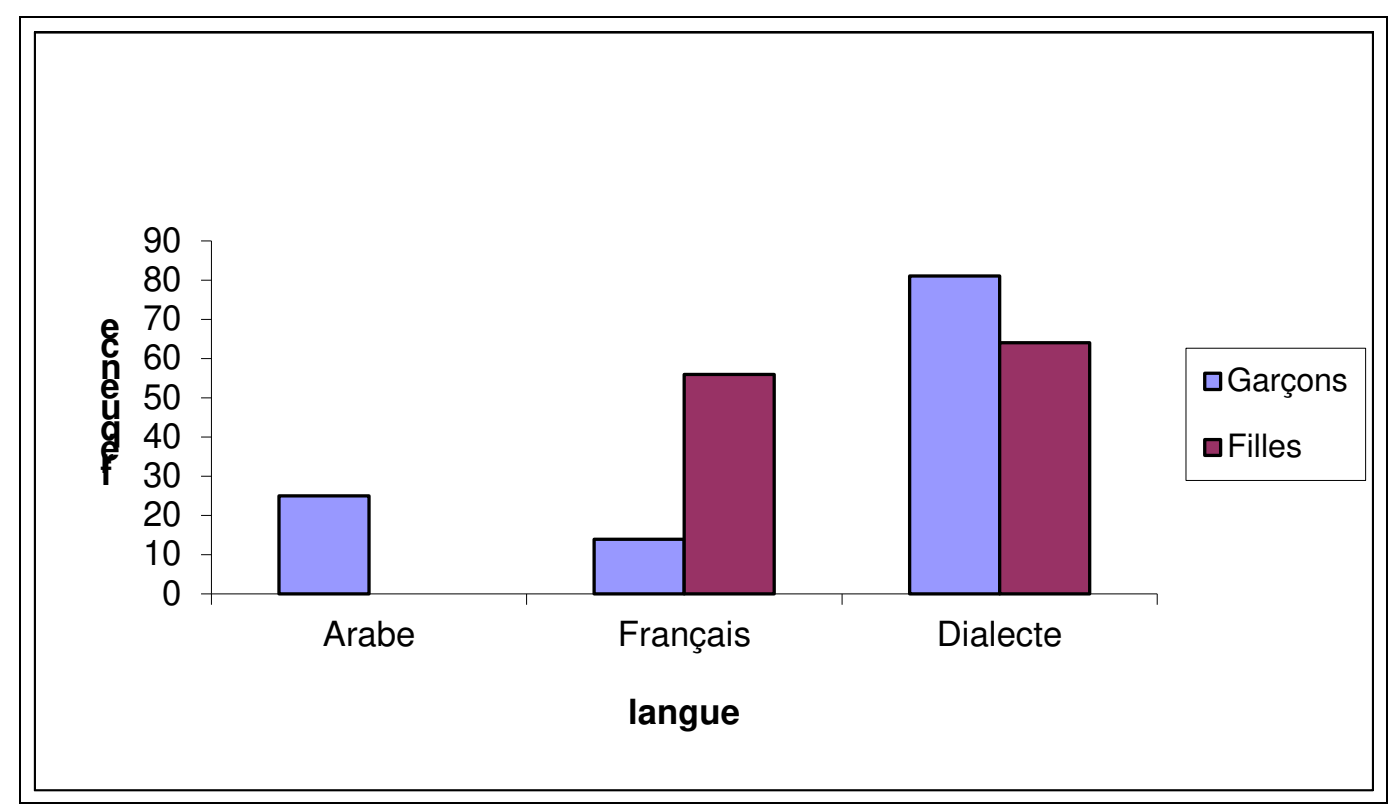

Figure 1 : saut de l'ange

$X_{2 G}=91,88 \quad$ TS à $P<0.001$

2. 2. Renversé avant

Afin de mesurer jusqu'à quel point le choix de la langue est influencé par les connaissances antérieures de l'élément gymnique, nous leur avons proposé le renversé 
avant et le renversé arrière. Le choix du dialecte est de $45 \%$ pour le renversé avant. Nous notons que le français est de $47 \%$, alors que la langue formelle est de $7,9 \%$.

Tableau II : renversé avant

Fréquence

\begin{tabular}{llll}
\hline & Arabe & Français & Dialecte \\
\hline Garçons & 13 & 50 & 57 \\
Filles & 6 & 63 & 51 \\
\hline
\end{tabular}

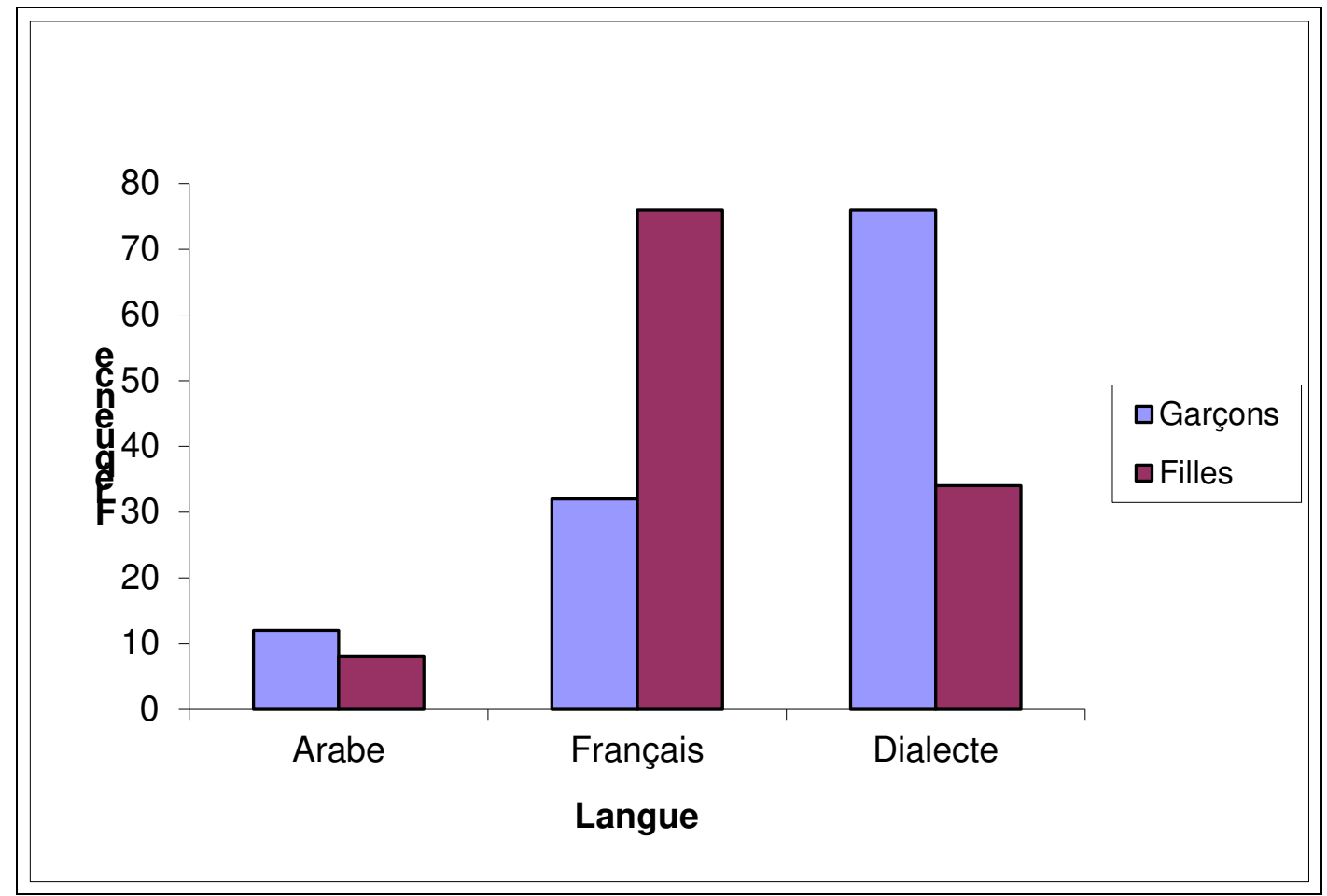

Figure 2 : Renversé avant

\section{$\underline{X_{2 G}}=69,93 \mathrm{TS}$ à $P<0.001$}

\section{3. Renversé arrière}

La comparaison des choix de la langue montre que le dialecte a une valeur de 46,2\% alors que la langue française est de $45 \%$. La langue arabe (formelle) se place loin derrière avec un pourcentage de $8,4 \%$. 
Tableau III : Renversé arrière

\section{Fréquence}

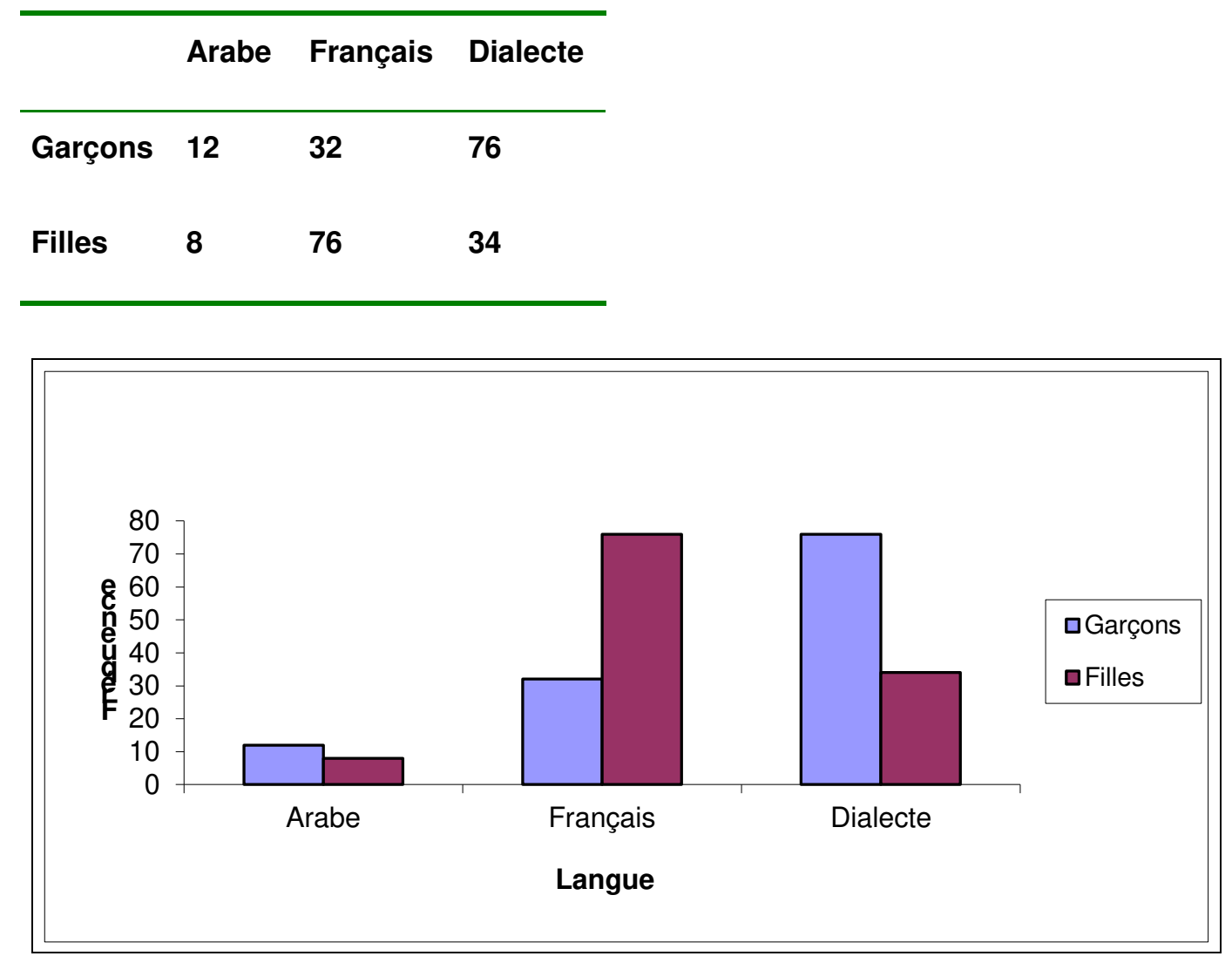

Figure 3 : Renversé arrière

$X_{2 G}=66,59$ TS à $P<0.001$

2. 4. ATR

Tableau IV : ATR

Fréquence

\begin{tabular}{llll}
\hline & Arabe & Français & Dialecte \\
\hline Garçons & 12 & 52 & 56 \\
Filles & 6 & 73 & 41 \\
\hline
\end{tabular}




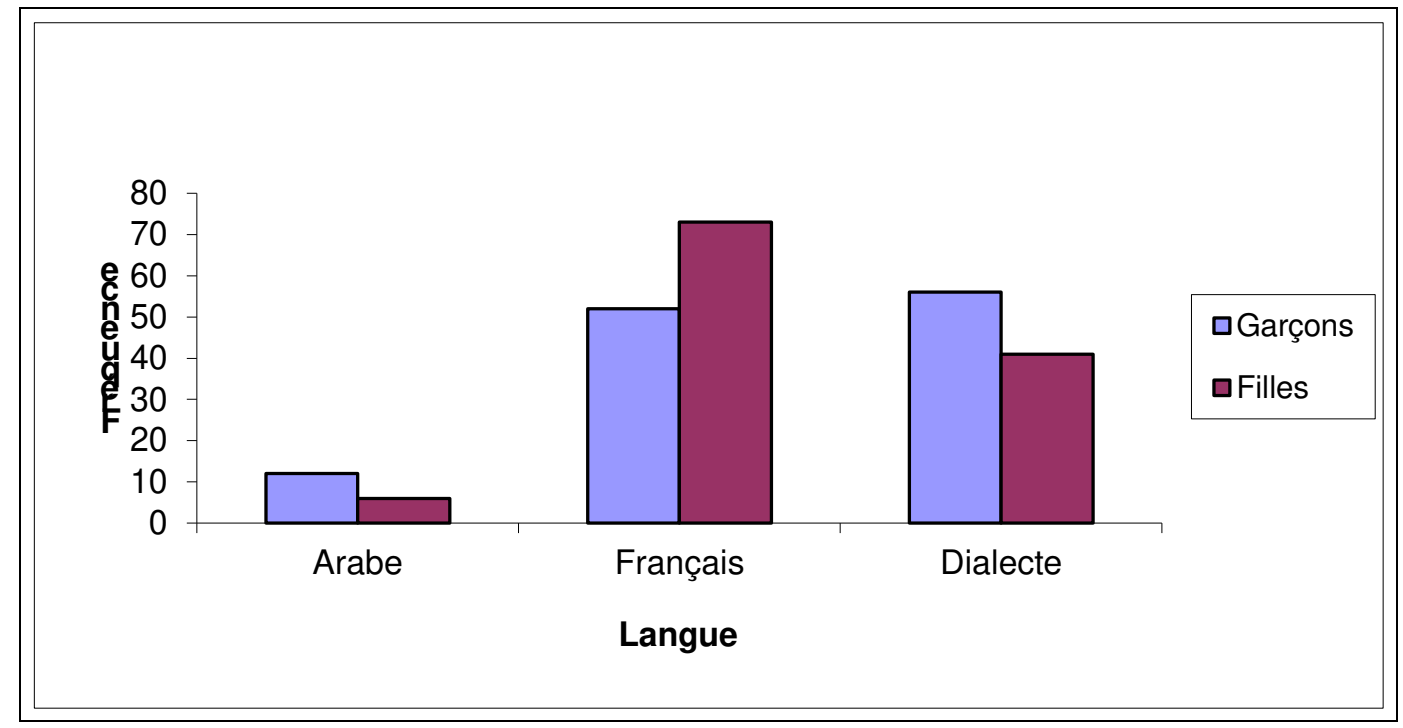

Figure 4 : ATR

$X_{2 G}=76,98$ TS à $P<0.001$

L'ATR est l'élément gymnique le plus enseigné dans le système scolaire tunisien. L'enseignant élabore plusieurs stratégies au cours des séances pour que l'élève puisse le maîtriser. L'explication et les démonstrations occupent une place importante dans le processus d'apprentissage car il est l'élément clé dans l'apprentissage en gymnastique. L'alignement des segments avec l'équilibre en position renversée est une habileté motrice fondamentale en gymnastique. Le pourcentage de $52,08 \%$ de réponse en langue française est très significatif, alors que l'arabe n'a q'une pourcentage de $7,5 \%$. La deuxième langue d'expression est le dialecte avec un pourcentage de 40,41\%. L'analyse des réponses montre que l'appui tendu renversé est dit « équilibre sur les mains " en français et « équilibre ala lidin » en dialecte.

2. 5. Renversé avant passage à l'ATR

Tableau $V$ : Renversé avant passage à l'ATR

Fréquence

\section{Arabe Français Dialecte}

Garçons $12 \quad 52 \quad 56$

$\begin{array}{llll}\text { Filles } & 6 & 73 & 41\end{array}$ 


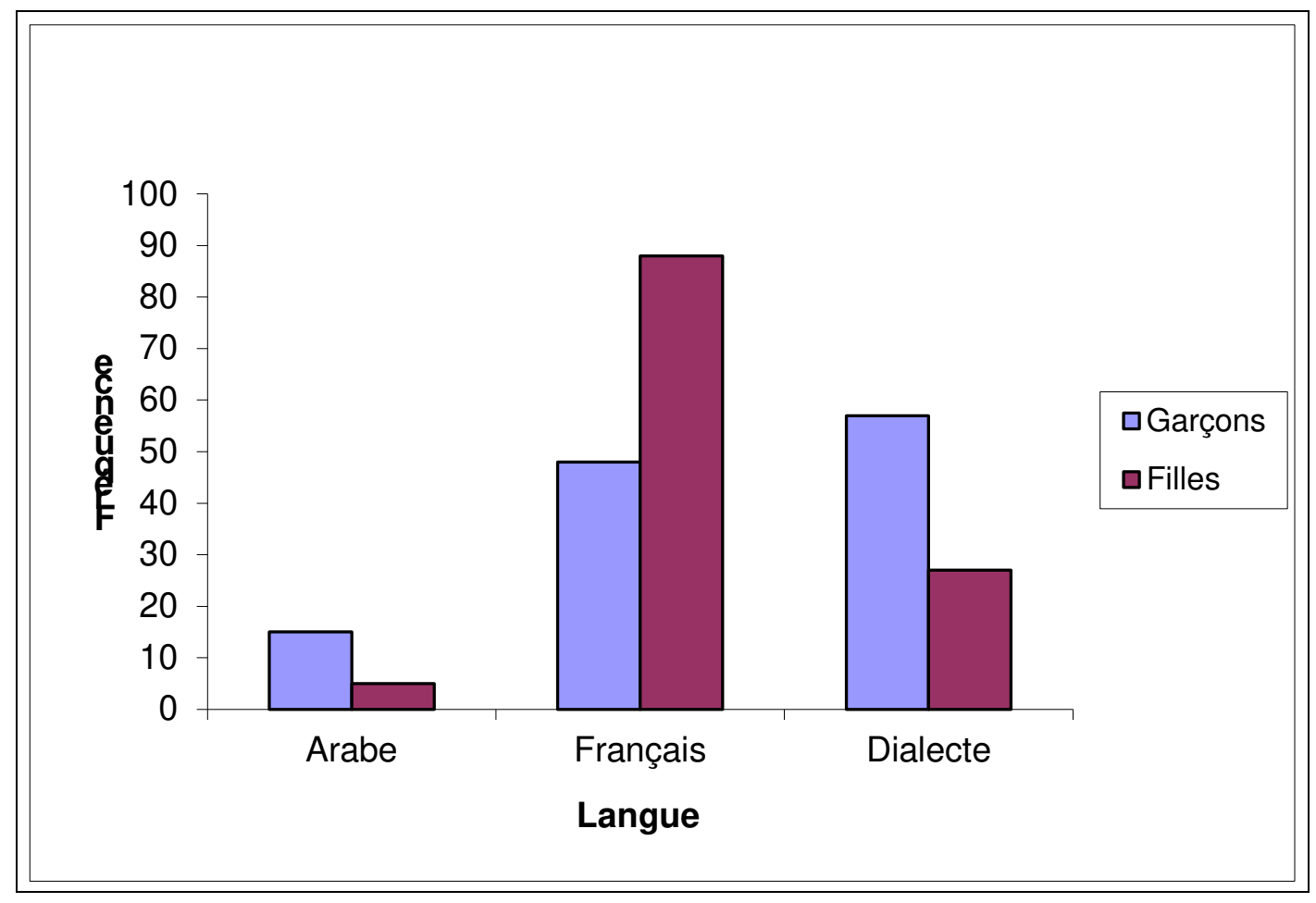

Figure 5 : Renversé avant passage à l'ATR

$X_{2 G}=84,4$ TS à $P<0.001$

Le français occupe la première place avec un pourcentage de $56,6 \%$ dont $73,3 \%$ des filles et $35 \%$ des garçons. Le renversé avant passage à l'ATR est la liaison de deux éléments gymniques déjà identifiés auparavant par les élèves.

\section{6. ATR Jambes serrées pont}

L'ATR jambes serrés pont fait partie des éléments gymniques nouveaux proposés aux élèves. Le choix de notre population d'étude est porté sur la langue française avec un pourcentage de $76,5 \%$, avec un pourcentage de $89 \%$ chez les filles et $65 \%$ chez les garçons ce qui explique l'importance des échanges en langue française. Ceci explique notre hypothèse de départ que la langue informelle est une langue d'énonciation et d'interaction. 
Tableau VI : ATR Jambes serrées pont

\section{Fréquence}

\begin{tabular}{llll}
\hline & Arabe & Français & Dialecte \\
\hline Garçons & 2 & 98 & 10 \\
Filles & 11 & 78 & 31 \\
\hline
\end{tabular}

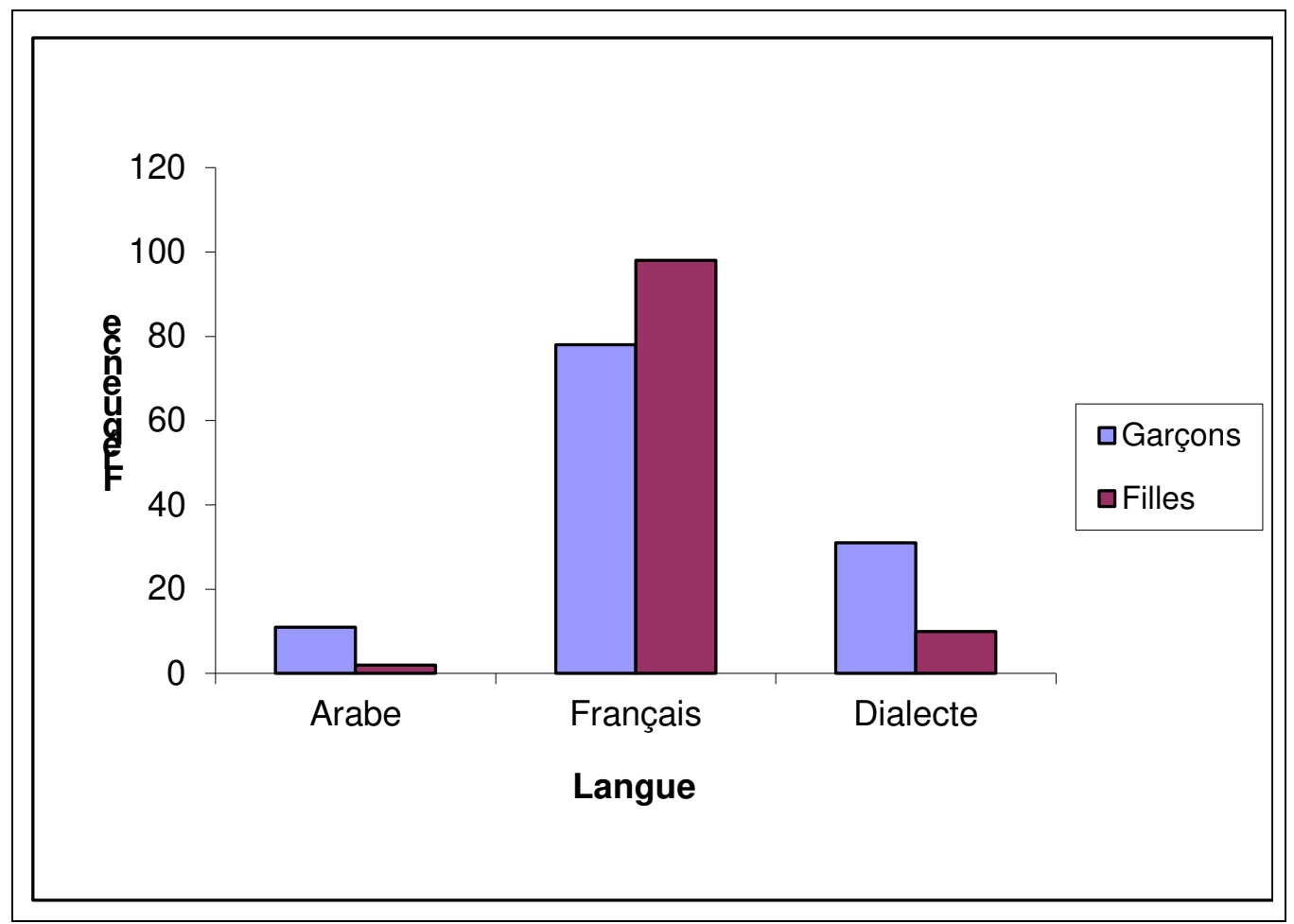

$X_{2 G}=198,17$ TS à $P<0.001$

Figure 6 : ATR Jambes serrées pont

\section{7. ATR Valse -tour}

La tendance à utiliser la langue française est la plus importante dans notre recherche. $83,3 \%$ des élèves se sont exprimés en français dont $84,1 \%$ de filles et $82,5 \%$ de garçons. Si l'ATR est facilement identifiable par les élèves nous croyons que le mot « valse " pose un vrai problème de traduction. Culturellement la danse " valse » est étrangère à la danse tunisienne. Ce seul lexique montre qu'un véritable processus de francisation linguistique est véhiculé par la culture scolaire. L'introduction d'un nouvel élément gymnique se fait par le biais de la langue de l'enseignant. Donc le terme véhicule les règles et les pratiques importées de l'occident. 
Tableau VII : ATR Valse -tour

\section{Fréquence}

\section{Arabe Français Dialecte}

\begin{tabular}{llcc}
\hline Garçons & 8 & 99 & 13 \\
Filles & 5 & 101 & 14 \\
\hline
\end{tabular}

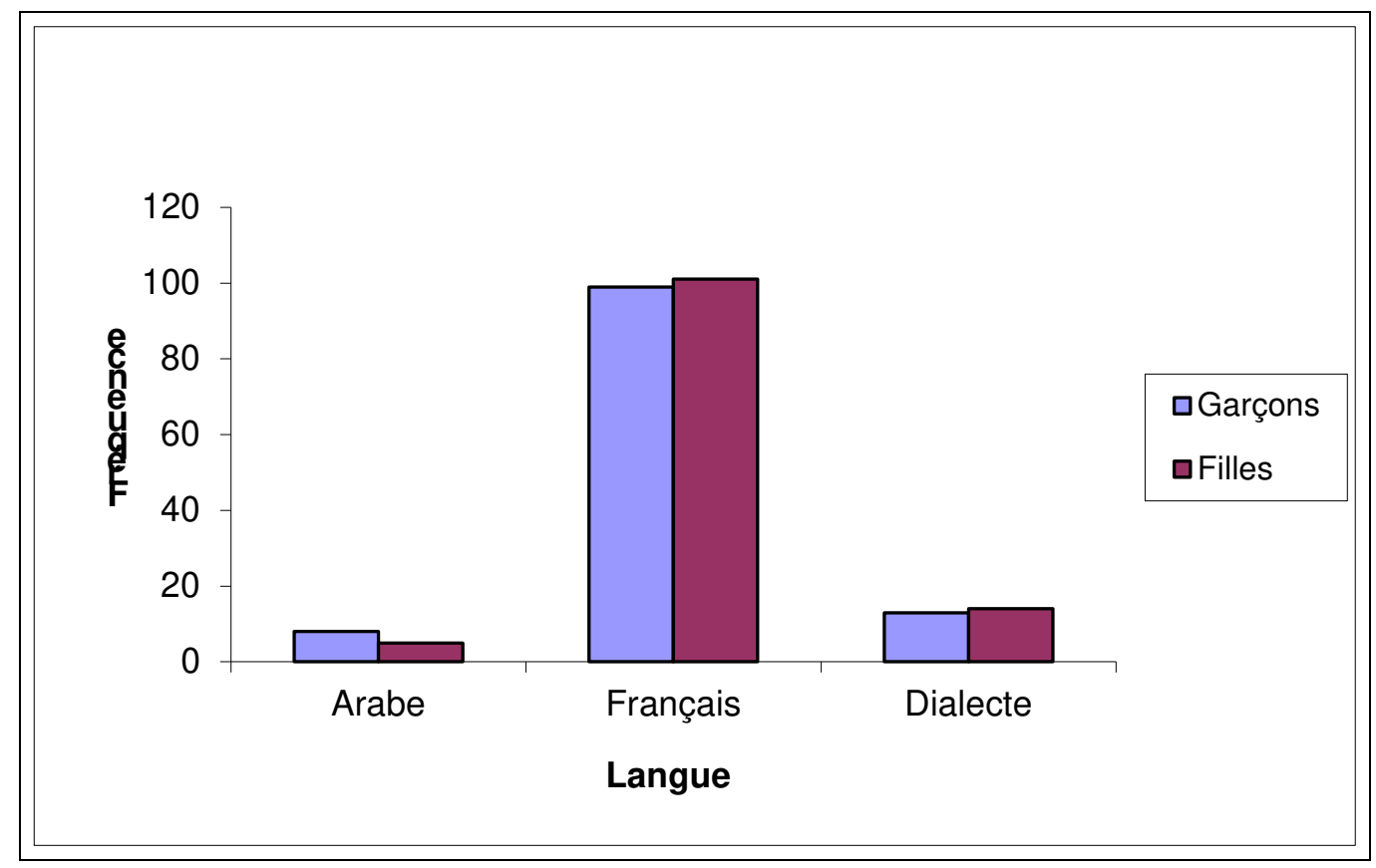

Figure 7 : ATR Valse -tour

$X_{2 G}=271,23$ TS à $P<0.001$

\section{8. Rondade saut vertical}

Le pourcentage de l'utilisation de la langue française est de 69,5\%. L'analyse du contenu pointe la difficulté de la traduction du mot saut «kafz ». Dans le langage sportif tunisien le mot saut est identifié en tant que saut vers l'avant et non pas vers le haut alors que la rondade est différente de la roulade : «tkarbissa » en dialecte et « dahraja » en arabe et de renversé : « tchakliba » en dialecte. 
Tableau VIII : Rondade saut vertical

\section{Fréquence}

\begin{tabular}{llll}
\hline & Arabe & Français & Dialecte \\
\hline Garçons & 16 & 85 & 19 \\
Filles & 3 & 75 & 32 \\
\hline
\end{tabular}

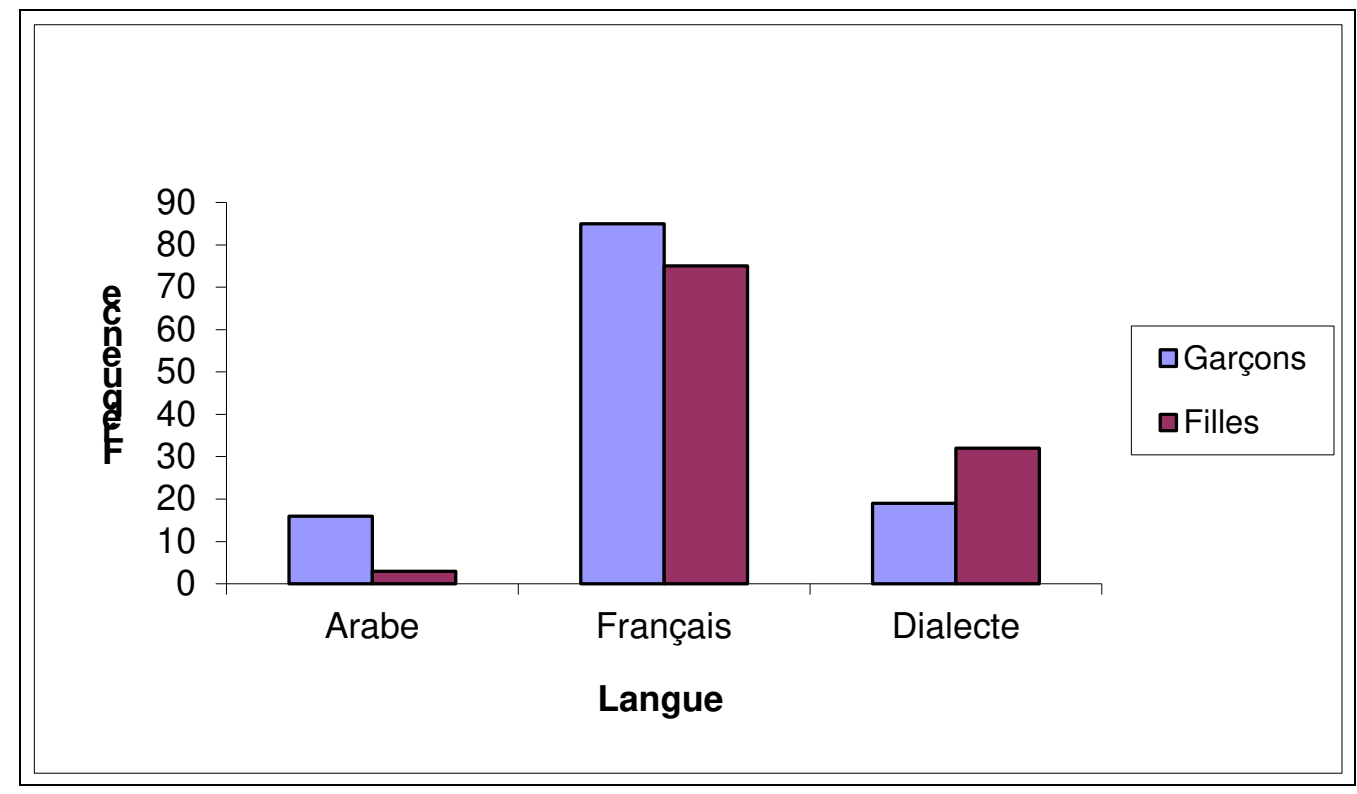

$X_{2 G}=142,55$ TS à $P<0.001$

Figure 8 : rondade saut vertical

\section{Discussions et interprétations}

Les résultats obtenus sont à nuancer compte tenu, d'une part des nombreuses sources de biais qu'il est impossible de contrôler et d'autre part de l'effectif restreint examiné.

Nous pouvons dire à l'issue de l'examen des réponses fournies par les élèves qu'il existe une différence très significative entre le choix des deux langues informelles (français et dialecte) d'une part et de la langue formelle (arabe) d'autre part. Les résultats obtenus dans cette recherche tendent à confirmer globalement que l'interaction langagière informelle est plus importante dans une séance d'éducation physique. L'arabisation du système scolaire tunisien (manuel, langue d'enseignement, circulaires) a commencé pendant les années 80. La langue arabe est la langue officielle d'enseignement. Nous pouvons affirmer que, dans le cadre de l'enseignement des activités gymniques, les élèves communiquent, en privilégiant du moins entre eux et avec l'enseignant le français ou le dialecte ce qui laisse dire que l'acquisition et l'intériorisation lors de l'apprentissage 
sont facilitées par l'interaction langagière informelle. L'enseignant qui s'engage dans cette perspective doit nécessairement réaliser une analyse logique préalable du moyen de communication efficace qui lui permet d'atteindre son objectif.

Notre recherche confirme les résultats de la littérature concernant la double fonction de la communication: une fonction de communication externe utilisée pour faciliter la compréhension des tâches à travers les échanges verbaux entre les membres du groupe et une fonction de communication «interne à l'individu» lui permettant d'intérioriser la tâche. Cette communication interne constitue le langage égocentrique (Galperine, 1966, Vygotsky, 1985), qui n'est autre qu'une manifestation du passage d'un fonctionnement inter-psychique à un fonctionnement intra-psychique: c'est le passage d'une activité sociale et collective à une activité plus individualisée.

Le dialecte qui est la forme individualisante du langage (franco-tunisien) se conforme à des règles sociales. II est valorisé comme expression masculine. Par contre, l'expression en langue française est une forme de socialisation des filles. Comme le montre nettement notre recherche, elles préfèrent et maîtrisent mieux la langue française que les autres moyens de communication langagière. La différence des sexes, si elle procède d'une «nécessité biologique », est aussi un principe universel d'organisation sociale. Un des objets de cette recherche, en terme de genre, est de fournir des éléments d'analyse des exigences culturelles de l'école. Le système des savoirs scolaires ne repose sur aucune justification objective. II est jugé digne d'être transmis par la langue formelle d'enseignement. L'interaction langagière dans une séance d'éducation physique varie selon les publics d'élèves.

\section{En guise de conclusion}

Associant approche historique, questionnement épistémologique et approches expérimentales, la démarche proposée s'est donnée pour but de montrer qu'une approche sociale qui tient compte de la langue informelle est une importante contribution au développement d'une forme d'intelligence que l'école a souvent négligée faute de l'avoir définie et étudiée.

Ainsi, cette approche permet-elle de rendre pertinente l'éducation physique et sportive en réintégrant une nouvelle forme de communication verbale, le dialecte. En effet, la langue officielle (arabe) si souvent favorisée dans le milieu scolaire, ne reflète qu'un aspect, aussi important soit-il, de la communication. En d'autres termes, une place doit être faite dans l'enseignement de l'éducation physique et sportive aux autres formes d'interaction langagière. 
Ceci nous autorise à dire que, peut-être, les modes de communications langagières informelles en éducation physique et sportive (français et dialecte), considérées comme perturbatrices pour les apprentissages et souvent passés sous silence dans le cadre de l'enseignement, sont les plus importantes. Ainsi, la construction de savoirs concernant la communication reconnaîtrait la spécificité de l'interaction langagière en éducation physique et sportive. Cette reconnaissance pourrait permettre un attrait plus important pour la pratique. Cette recherche nous met en garde contre un certain nombre d'illusions ou d'idées reçues concernant l'enseignement de l'EPS: illusion de l'existence d'une langue commune pour l'EPS, illusion de la spécificité de cette discipline et enfin illusion d'une cohérence entre discours sur les principes et discours sur les pratiques. Entre langue formelle et langue informelle, I'EPS aurait-elle implicitement opté pour cette dernière ? La prise en compte des résultats de notre recherche appuie cette constatation. Nous sommes invités à nous interroger sur cette spécificité d'interaction langagière en EPS. II convient de souligner que dans notre recherche il y a deux moments forts de l'interaction langagière informelle. Pour le premier moment, il s'agit d'identifier des éléments déjà vus lors des cycles précédents en gymnastique. Nous constatons que le dialecte est la langue la plus choisie par les élèves. L'école n'est pas la seule institution tunisienne dont le dialecte est le moyen principal de communication. Les pages sportives de quelques journaux ainsi que les commentaires sportifs dans les radios privées véhiculent cette orientation. Pour le second, il s'agit d'identifier des éléments totalement nouveaux. Le français est la langue dominante dans le choix des élèves. L'usage de cette langue peut s'expliquer d'un côté par l'influence de l'enseignant et de l'autre par l'incapacité de la langue officielle (arabe) à intégrer des pratiques corporelles étrangères à la culture tunisienne (valse). Comprendre ce paradoxe nécessite d'étudier les conflits dont le système d'enseignement est le lieu et l'enjeu. L'arabisation du système scolaire tunisien est entamée depuis vingt ans. La langue arabe prend la place du français (langue des colons). Ayant à l'esprit ce qui se dégageait de l'analyse statistique de notre échantillon cela semble vouloir dire que l'interaction langagière en arabe est pratiquement absente, et que la langue d'enseignement est le français. Cette situation est liée à un état de rapport pédagogique, à un état des rapports entre le système d'enseignement et ce qu'on appelle la société globale, comme le soulignait Bourdieu (1993, p. 96). 


\section{Bibliographie}

Belaid, H. (1995), "Les sociétés françaises de tir et de préparation militaire dans la Tunisie coloniale au cours des années vingt » in Revue des Sciences Humaines ("Les cahiers de Tunisie "), Tome XLVIII, n spécial 169-170, $2^{\text {ème }}$ et $3^{\text {ème }}$ Trimestre.

Blaye, A. (1987). Organisation du produit de deux ensembles: influence des interactions sociales entre pairs sur les procédures de résolution et les performances individuelles. European journal of éducation,1,4, 29-44.

Bronckart, J.P. \& Schneuwly, B. (1985). Vygotsky aujourd'hui. Paris: Neuchâtel, Delachaux et Nestlé.

Bourdieu. P. (1993), Questions de sociologie, Tunis, Cérs Editions.

Collard, L. (2005). Quels transferts entre gymnastique et natation ?. Revue EPS, 314, 1114.

Crahay, M., Lafontaine, D., (1986), L'art et la science de l'enseignement, Belgique, Editions Lamour.

Delignières, D. (1992). Apprentissage moteur et verbalisation. Echanges et contreverses, 9-41.

Deriaz, D., Poussin. B., Gréhaigne, J.F., (1998). Le débat d'idées. Education Physique et Sport, 273, 80-82.

Dugas, E. (2004). Education physique et éducation informelle à l'école . Revue Education et Société, 10, 21-34.

During, B. (2000). Histoire Culturelle des Activités Physiques. Paris, Vigot.

During, B. (2005). L'éducation physique une discipline en progrès? Carrefour de l'éducation, 20.

Gal-Petiffaux, N. \& Cizeron M. (2006), Motifs « cachés » et motifs «apparents » de la donation de parole aux élèves en classe : étude de cas d'enseignants novices en éducation physique in Intervenir en éducation physique et en sport : recherches actuelles, coordonné par Carlier G, Bouthier D et Bui'Xuân G, Presses Universitaires de Louvain.

Gréhaigne, J.F., \& Cadopi., M., (1990). Apprendre en éducation physique, in Education physique et didactique des APS, pp. 17-24, Paris : AEEPS.

Orléon, P. (1978). Langage et développement mental. Pierre Margada, éditeur.

Parlebas, P. (1999). Jeux, sports et sociétés. Lexique de praxéologie motrice, Paris, INSEP Publications. 
Payen, P. (1998). L'apport du langage à l'apprentissage d'une action dynamique en E.P.S. Dossier E P.S N³9. p. 65-69. Paris : Revue E. P. S.

Perret-Clermont, A.N. (1981). La construction de l'intelligence dans l'interaction sociale. Berne : Peter Lang.

Vygotsky, L. (1985). Pensée et langage. Paris : Editions sociales. 